\title{
NUTRITION DEFICIENCY RISK ASSESSMENT OF FREE-LIVING OLDER ADULTS IN SINGAPORE
}

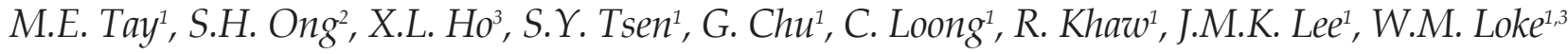

\begin{abstract}
Background: The need to identify those at risk of nutrition deficiency is critical in promoting good nutritional status in older adults. Objectives: The study aims to assess the risk of nutrition deficiency of free living, older adults in Singapore. Design, Setting and Participants: Free-living adults (aged 50 and above) were recruited from various community centres under the supervision of the Peoples' Association of Singapore. Measurements: Nutrition deficiency risk assessment was performed with all participants in person using the validated 15-item Seniors in the Community Risk Evaluation for Eating and Nutrition II (SCREEN II) tool. Anthropometric assessments were also conducted. Results: Majority $(88.1 \%)$ of the one hundred ninety three participants (83.9\% women, $66.8 \pm 8.3$ years of age) showed risk of nutrition deficiency. $68.4 \%$ of the participants fell under the "high risk" group. The women showed higher risk of nutrition deficiency than the men in the study group. Less than $12 \%$ of the participants consumed five or more servings of vegetables and fruits in a day, and approximately $25 \%$ ingested less than two servings per day. Almost half of the participants consumed dairy products less than once a day. Less than $20 \%$ of the participants consumed more than 2 servings of dietary protein source daily. More than $60 \%$ of the participants were overweight. Conclusions: A significant proportion of free-living older adults in Singapore community is experiencing high risk of nutrition deficiency as measured by the SCREEN II. The same older adults are overweight and thereby are exposed to the elevated health risk associated with obesity.
\end{abstract}

Key words: Nutrition deficiency, older adults, food choice, obesity.

\section{Introduction}

The Singaporean population is tending to live much longer (with one of the highest life expectancies for both men and women worldwide) (1). At the same time as individuals are living longer throughout the world, they also seem to be experiencing a lower number of Healthy Life Years (i.e. lifespan spent in "good health") (1). Dietary habit is generally accepted to be strongly associated with risk of most major disease as a result of large, population-based studies from around the world $(2,3)$. A number of studies in Europe and the United States had assessed the nutritional status of older population groups $(4,5)$. Singapore has a unique food culture and geography compared to other high income developed countries where previous studies have been carried out. Recent prospective dietary studies examining the relations between dietary intake and health outcomes

1. Food Science \& Nutrition Group, School of Chemical \& Life Sciences, Nanyang Polytechnic, Singapore; 2. Acumen Research Laboratories Pte Ltd, Diagnostics Development Hub, Biopolis, Singapore; 3. Centre for Functional Food \& Human Nutrition, School of Chemical \& Life Sciences, Nanyang Polytechnic, Singapore.

Corresponding Author: Dr Wai Mun Loke, Centre for Functional Food \& Human Nutrition, School of Chemical \& Life Sciences, Nanyang Polytechnic, 180 Ang Mo Kio Ave 8, Singapore, Email: loke_wai_mun@nyp.edu.sg, Fax: +65 65520844 in a specific group within the Singapore population did not provide sufficient data on the nutritional status within an "at-risk" group such as older adults (6-8). Older adults are currently one of the most vulnerable group to malnutrition and yet this nutrition problem continues to be unrecognized and untreated (9). The problem may arise from many factors, including those of social, economic and epidemiologic context. Nutrition deficiency prevalence in the western community has been reported to vary from 10-30\% (10), highlighting the significance of nutrition deficiency, particularly in the community-dwelling older adult population. Nutrition risk screening is a process of identify characteristics known to be associated with dietary or nutritional problems (5). Identifying those at risk of nutrition deficiency is critical in providing optimal care and promoting good nutritional status in communitydwelling older adults (5). Screening the older adults for nutrition deficiency risk would assist the policy makers to elucidate possible reasons for malnutrition, implement critical nutrition policies to reduce the nutrition deficiency risk, and thereby prevent the incidence of nutrition deficiency in these older adults. There are currently limited data on the nutrition deficiency risk of community-living older adults in Singapore.

Anthropometry involves obtaining physical 
measurements of an individual, then relating them to standards that reflect the growth and development of that individual. It has been routinely performed as an essential part of nutrition assessment useful for evaluating malnutrition (11). Currently, there is limited data to associate nutrition deficiency risk and anthropometric parameters of older adults.

Our study aimed to assess and evaluate the nutrition deficiency risk of free-living, older adults in Singapore. The nutrition status of these older adults was examined using inexpensive and non-invasive anthropometric measurements. The same study also examined the possible associations between body physique and nutrition deficiency risk in these older adults.

\section{Methods}

\section{Study participants and Setting}

The study was approved by the Institutional Review Board, Nanyang Polytechnic. Free-living adults (aged 50 and above) were recruited from various community centres managed by the People's Association of Singapore. The age criterion of 50 years old and above had been selected as to capture the nutrition deficiency risk of these individuals entering into their retirement years. Implied informed consent was obtained from each participant. Exclusion criteria included individuals living within a care-home or sheltered accommodation setting, those who are housebound or have been clinically diagnosed as being cognitively impaired. Demographic information, including age, gender, education years, marital status and living conditions, was obtained from the participants.

\section{Nutrition Deficiency Risk Assessment}

Nutrition deficiency risk assessment was performed using the validated SCREEN II (Seniors in the Community Risk Evaluation for Eating and Nutrition II) tool $(12,13)$. SCREEN II is a validated nutrition screening tool that caters to older adults. The tool comprises of 14-item questionnaire on nutrition risk factors influencing the older adults. These factors include appetite, frequency of eating, motivation to cook, ability to shop and prepare food, weight changes, isolation and loneliness, chewing and swallowing, digestion and food restrictions. It was conducted with all participants in person. Taken together, the tool helps to determine if an older adult has a potential nutritional problem or is at risk of developing one and identifies those who need further nutrition assessment and treatment. SCREEN II tool has been validated to assess the nutritional risk level of older adults in order to identify those at risk of nutrition deficiency $(12,13)$. The questionnaire was individually administered to each participant during a face-to-face interview. Trained interviewers asked the questions and completed the forms. The SCREEN II score of the participant was calculated and compared to the scoring guide to determine if the participant was at risk of nutrition deficiency. The SCREEN II scoring is categorized as follows: low risk (score $\geq 54$ ), moderate risk $(50 \geq,<54)$, high risk $(<50)$. The lower the score, the greater is the nutrition deficiency risk. The areas where nutrition could be improved would be identified for those participants at risk.

\section{Anthropometric Measurement}

Anthropometric measurements, including weight, height, waist circumference, hip circumference, mid arm muscle circumference, calf circumference, triceps skinfold and body composition were performed. Body weight and height were measured using weighing balances (Omron, JAPAN) and stadiometers (Seca, GERMANY). Waist, hip, mid arm and calf circumferences were measured, using flexible, non-stretching measuring tapes, as the circumference of the waist (approximately on the level of the umbilicus), the largest circumference of the hip, the circumference along the midpoint of the arm between the shoulder and elbow tips, and the largest circumference of the calf, respectively. The triceps skinfold - the width of a fold of skin taken over the triceps muscle - was measured using the skinfold calipers. Body composition was measured using bioelectrical impedance body composition analyser (ioi 352, Jawon, SOUTH KOREA). The SCREEN II tool and anthropometric measurements were completed during the same visit to the study centre. The measured body mass index, waist circumference and waist-hip circumference ratio were stratified according to the health risk levels from the Singapore Health Promotion Board.

\section{Statistical Analyses}

Statistical analyses were performed using SPSS version 22.0 (SPSS Inc., Chicago, IL, USA). Data were presented as mean \pm standard deviation (SD). Between-group (men vs women) differences were analyzed using unpaired t-tests. Correlations between anthropometric parameters were analyzed using Pearson's correlation test. Statistical significance was set at $\mathrm{p}<0.05$.

\section{Results}

\section{Participant Characteristics}

One hundred and ninety-three participants (83.9\% women, $66.8 \pm 8.3$ years of age) were recruited. The men were significantly older than the women $(p<0.05$ using unpaired t-test, Table 1). Majority of the participants had at least six years of formal school education and was 
Table 1

SCREEN II risk scores, demographic and anthropometric characteristics of 193 study participants by gender and overall sample

\begin{tabular}{|c|c|c|c|}
\hline & Overall & Male & Female \\
\hline \multicolumn{4}{|l|}{ Demographic Characteristics } \\
\hline Age (years) & $66.8 \pm 8.3$ & $69.7 \pm 8.7$ & $66.2 \pm 8.1^{*}$ \\
\hline Gender, female (\%) & 83.9 & - & - \\
\hline SCREEN II risk score & $46.4 \pm 5.9$ & $49.1 \pm 4.6$ & $45.9 \pm 6.0^{*}$ \\
\hline Marital status, married (\%) & 99.5 & 100.0 & 99.4 \\
\hline Education, $>6$ years formal education $(\%)$ & 86.4 & 74.2 & 88.8 \\
\hline Living with family / friend (\%) & 90.0 & 96.7 & 88.7 \\
\hline Living in owned house $(\%)$ & 98.4 & 100.0 & 98.1 \\
\hline Medical visit in past 3 months (\%) & 51.0 & 51.6 & 50.9 \\
\hline \multicolumn{4}{|l|}{ Anthropometric Characteristics } \\
\hline Weight $(\mathrm{kg})$ & $56.7 \pm 9.4$ & $62.3 \pm 11.0$ & $55.7 \pm 8.7^{*}$ \\
\hline Height (m) & $1.55 \pm 0.06$ & $1.62 \pm 0.05$ & $1.54 \pm 0.06^{*}$ \\
\hline Body mass index (kg m-2) & $23.6 \pm 3.5$ & $23.5 \pm 3.3$ & $23.6 \pm 3.6$ \\
\hline Waist Circumference (cm) & $85.5 \pm 12.9$ & $89.8 \pm 9.7$ & $84.7 \pm 13.3^{*}$ \\
\hline Hip circumference $(\mathrm{cm})$ & $94.6 \pm 12.7$ & $95.2 \pm 8.3$ & $94.5 \pm 13.4$ \\
\hline Waist-Hip Ratio & $0.91 \pm 0.10$ & $0.95 \pm 0.09$ & $0.90 \pm 0.10^{*}$ \\
\hline Triceps Skinfold (cm) & $19.5 \pm 7.0$ & $14.0 \pm 5.4$ & $20.5 \pm 6.8^{*}$ \\
\hline Midarm circumference (mm) & $279 \pm 45$ & $280 \pm 30$ & $279 \pm 47$ \\
\hline Calf circumference $(\mathrm{cm})$ & $34.2 \pm 6.1$ & $35.3 \pm 3.1$ & $34.0 \pm 6.5$ \\
\hline Body Fat Mass (\%) & $30.3 \pm 8.5$ & $20.1 \pm 6.2$ & $32.1 \pm 7.5^{*}$ \\
\hline
\end{tabular}

${ }^{*} \mathrm{p}<0.05$ vs male participants using unpaired t-test.

living with spouses or family members (Table 1). Almost all participants were married, and were residing in owned houses (Table 1). Close to half of the participants required medical attention in the three months prior to the study (Table 1).

\section{Risk of Nutrition Deficiency}

The participants showed risk of nutrition deficiency as indicated by the mean overall SCREEN II score (46.4), which is lower than the validated overall low-risk score (54.0) (Table 1) $(12,13) .88 .1 \%$ of the participants had scores below the validated low-risk score (Table 2). 68.4\% of the participants scored less than 50 and fell under the "high risk" group (Table 2) (12, 13). The women showed significantly higher risk of nutrition deficiency than the men in the study group (men, $49.1 \pm 4.6$ vs women, 45.9 \pm 6.0 ; $\mathrm{p}<0.05$ using unpaired t-test; Table 1). SCREEN II items with a score of 2 or less indicates potential for nutrition risk $(12,13)$. From the SCREEN II score breakdown, the participants did better for recent body weight change, weight perception, not skipping meals, not limit or avoid certain food, meal preparation, eating / chewing difficulty and groceries shopping (Table
3). $39.8 \%$ of the participants thought that their weights were not ideal, but less than $4 \%$ had unintentional weight changes (Table 3). Near $6 \%$ of the participants skipped their meals frequently and approximately $40 \%$ limited or avoided certain foods in their diet (Table 3). Close to75\% of the participants prepared their own meals frequently or were satisfied with the quality of their diets with less than $6 \%$ having difficulty chewing their food or with their groceries shopping (Table 3). Nearly $80 \%$ of the participants reported good to very good appetite during meals, and a fair proportion of them $(\sim 60 \%)$ had regular meals with someone (Table 3 ). The participants did not score well with their dietary choice (Table 3). The studied participants consumed less than sufficient amounts of fruits, vegetables and dairy products. Less than $12 \%$ of the participants consumed five or more servings of vegetables and fruits in a day, and approximately $25 \%$ ingested less than two servings per day (Table 3 ). Almost half of the participants consumed dairy products less than once a day (Table 3). Less than $20 \%$ of the participants consumed more than 2 servings of dietary protein source daily (Table 3).

The SCREEN II scores were not statistically correlated to age, years of formal education and other measures of 
Table 2

Frequency Distribution of SCREEN II Scores and Anthropometry - Health Indices in 193 older adult participants by gender and overall sample

\begin{tabular}{|c|c|c|c|c|c|c|}
\hline SCREEN II Score & \multicolumn{2}{|c|}{ Male* } & \multicolumn{2}{|c|}{ Female $^{*}$} & \multicolumn{2}{|c|}{ Overall +} \\
\hline Risk Level & Range & $\%$ & Range & $\%$ & Range & $\%$ \\
\hline High risk of nutrition deficiency & $<50$ & 54.8 & $<50$ & 71.0 & $<50$ & 68.4 \\
\hline Moderate risk & $50-53$ & 29.1 & $50-53$ & 17.9 & $50-53$ & 19.7 \\
\hline Low risk & $>54$ & 16.1 & $>54$ & 11.1 & $>54$ & 11.9 \\
\hline \multicolumn{7}{|l|}{ Body Mass Index $(\mathrm{kg} \mathrm{m}-2) \neq$} \\
\hline Risk Level & Range & $\%$ & Range & $\%$ & Range & $\%$ \\
\hline Risk of nutrition deficiency & $<18.5$ & 9.7 & $<18.5$ & 5.6 & $<18.5$ & 6.2 \\
\hline Low risk & $18.5-22.9$ & 25.8 & $18.5-22.9$ & 34.5 & $18.5-22.9$ & 33.2 \\
\hline Moderate risk & $23.0-27.4$ & 51.6 & $23.0-27.4$ & 45.7 & $23.0-27.4$ & 46.6 \\
\hline High risk & $\geq 27.5$ & 12.9 & $\geq 27.5$ & 14.2 & $\geq 27.5$ & 14.0 \\
\hline \multicolumn{7}{|l|}{ Waist Circumference $(\mathrm{cm}) \S$} \\
\hline Risk Level & Range & $\%$ & Range & $\%$ & Range & $\%$ \\
\hline Very low & $<80$ & 16.1 & $<70$ & 8.6 & - & - \\
\hline Low & $80-99$ & 71.0 & $70-89$ & 50.7 & - & - \\
\hline High & $100-120$ & 12.9 & $90-109$ & 40.7 & - & - \\
\hline Very high & $>120$ & 0 & $>110$ & 0 & - & - \\
\hline \multicolumn{7}{|l|}{ Waist-Hip Circumference Ratio I I } \\
\hline Risk Level & Range & $\%$ & Range & $\%$ & Range & $\%$ \\
\hline Excellent & $<0.85$ & 9.7 & $<0.75$ & 3.1 & - & - \\
\hline Good & $0.85-0.89$ & 12.9 & $0.75-0.79$ & 8.0 & - & - \\
\hline Average & $0.90-0.95$ & 45.1 & $0.80-0.86$ & 25.3 & - & - \\
\hline At risk & $>0.95$ & 32.3 & $>0.86$ & 63.6 & - & - \\
\hline
\end{tabular}

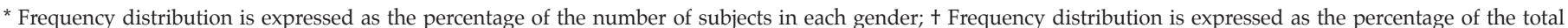

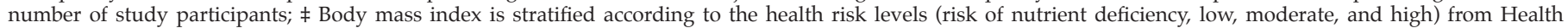

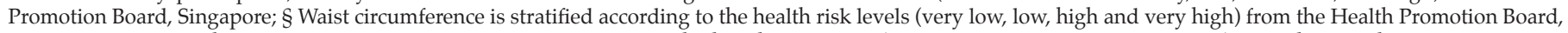

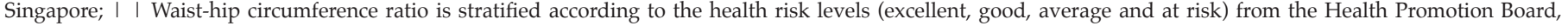
Singapore.

socioeconomic status.

\section{Anthropometry Characteristics}

The BMI did not differ between the genders although the male participants were significantly heavier and taller than the female counterparts (Table 1). The male participants had significantly larger waist circumferences and waist-hip circumference ratios, while the female participants had significantly larger triceps skinfolds and body fat compositions (Table 1).

The participants measured a mean BMI of 23.6, which falls in the moderate risk range. More than $60 \%$ of the participants were classified as overweight or obese, with their measured BMI falling into the moderate and high risk ranges $(\mathrm{BMI}>23.0)$ (Table 2). The observation was consistent across the genders as significant difference was absent in the distribution of BMI (Table 2). The male participants exhibited lower incidence of overweight than the female ones by comparing their waist circumferences and waist-hip circumference ratios (Table 2). Less than $10 \%$ of the participants were experiencing risks of nutrition deficiency according to their BMI (Table 2).

The body mass indices of the participants correlated significantly with the circumferences of their waists $(r=0.608)$, hips $(r=0.462)$, mid-arms $(r=0.586)$ and calfs $(r=0.396)$, triceps skinfolds $(r=0.492)$, body fat composition $(\mathrm{r}=0.727)$ and the calculated waist-hip circumference ratios $(r=0.290)$. The body fat masses of the participants associated significantly with BMI, waist $(\mathrm{r}=0.497)$, hip $(\mathrm{r}=0.465)$, mid-arm $(\mathrm{r}=0.531)$ and calf $(\mathrm{r}=0.305)$ circumferences, and waist-hip circumference ratio $(r=0.127)$. The correlations remained significant after gender stratification (data not shown). Significant 
Table 3

Cumulative Frequency of SCREEN II Score Items $(\mathrm{N}=193)$ by gender and overall sample

\begin{tabular}{|c|c|c|c|}
\hline \multirow[t]{2}{*}{ Score item } & \multicolumn{3}{|c|}{ Cumulative Frequency (\%) } \\
\hline & Overall & Male & Female \\
\hline \multicolumn{4}{|l|}{ Weight changed in the past 6 months } \\
\hline Don't know or weight changed & 19.3 & 14.8 & 20.3 \\
\hline Weight not changed & 100.0 & 100.0 & 100.00 \\
\hline \multicolumn{4}{|l|}{ Try to change weight in the past 6 months } \\
\hline No, but it changed anyway & 3.6 & 0.0 & 4.3 \\
\hline Yes or No & 100.0 & 100.0 & 100.0 \\
\hline \multicolumn{4}{|l|}{ You think your weight is } \\
\hline More or less than it should be & 39.8 & 25.8 & 42.5 \\
\hline Just right & 100.00 & 100.0 & 100.0 \\
\hline \multicolumn{4}{|l|}{ Skip meals } \\
\hline Almost everyday & 4.7 & 6.5 & 4.3 \\
\hline Often & 5.7 & 9.7 & 4.9 \\
\hline Sometimes & 24.4 & 19.4 & 25.3 \\
\hline Never or rarely & 100 & 100.0 & 100.0 \\
\hline \multicolumn{4}{|l|}{ Limit or avoid certain foods } \\
\hline Limit some food and find it difficult to manage & 3.1 & 6.5 & 2.5 \\
\hline Limit some food and managing fine & 42.0 & 25.8 & 43.8 \\
\hline Eat most food & 100.0 & 100.0 & 100.0 \\
\hline \multicolumn{4}{|l|}{ Describe your appetite } \\
\hline Poor & 1.6 & 0.0 & 1.9 \\
\hline Fair & 17.1 & 12.9 & 19.8 \\
\hline Good & 40.4 & 54.8 & 59.9 \\
\hline Very good & 100.0 & 100.0 & 100.0 \\
\hline \multicolumn{4}{|l|}{ Pieces or serving of fruit and vegetables eat per day } \\
\hline$<2$ & 24.4 & 16.1 & 25.9 \\
\hline 2 & 56.5 & 35.5 & 60.5 \\
\hline 3 & 73.1 & 54.8 & 76.5 \\
\hline 4 & 88.1 & 77.4 & 90.1 \\
\hline 5 or more & 100 & 100.0 & 100.0 \\
\hline \multicolumn{4}{|c|}{ Frequency of eating meat, eggs, fish, poultry, or meat alternatives per day } \\
\hline$<1$ & 12.4 & 3.2 & 14.2 \\
\hline 1 & 41.5 & 16.1 & 46.3 \\
\hline $1-2$ & 82.9 & 58.1 & 87.7 \\
\hline$>2$ & 100 & 100.0 & 100.0 \\
\hline \multicolumn{4}{|l|}{ Frequency of having milk products a day } \\
\hline$<1$ & 48.7 & 45.2 & 49.4 \\
\hline 1 & 79.8 & 83.9 & 79.0 \\
\hline $1-2$ & 94.3 & 93.5 & 94.4 \\
\hline $2-3$ & 99.5 & 100.0 & 99.4 \\
\hline$>3$ & 100.0 & 100.0 & 100.0 \\
\hline
\end{tabular}


Number of cups of fluid drank a day

$<2$

Cough, choke or have pain when swallowing food or fluids

Often or always

Sometimes

Never

Biting or Chewing difficulty

Often or always

Sometimes

Rarely

Never

100.0

100.0

Use of commercial meal replacements or supplements

Often or always

Sometimes

Never or rarely

100.0

100.0

100.0

Eat one or more meals a day with someone

Never or rarely

Sometimes

Often

Almost always

100.0

100.0

100.0

Statement best describes meal preparation for you

I usually find cooking a chore or I am not satisfied with the quality of food prepared by others

I sometimes find cooking a chore

I enjoy cooking most of my meals or I am satisfied with the

Problems getting groceries

Often or always

$\begin{array}{ccc}3.1 & 0.0 & 3.7 \\ 13.0 & 6.5 & 14.2 \\ 100.0 & 100.0 & 100.0\end{array}$

Sometimes

correlation was absent between the SCREEN II scores and the anthropometric measurements.

\section{Discussion}

Approximately $70 \%$ of the free-living older adults in Singapore recruited in our study were at high risk of nutrition deficiency as indicated by their SCREEN II scores. Fifty two percent of community-living, older adults $(n=108)$ was assessed with high risk of nutrition deficiency in a similar study conducted in New Zealand (14). In the same study, those with high risk tended to be widowed or living alone (14). The comparatively low number of widowed and living-alone participants in our study did not explain the observed high risk of nutrition deficiency. Our participants scored poorly in their food choice, especially the low intakes of fruits, vegetables and dairy products. Similar inadequate intakes of fruits, vegetables, and dairy products were highlighted in older adults from other communities. A study in the United States of America involving 420 community living older adults ( $>79$ year old) reported inadequate intakes of 4 or more nutrients in $80 \%$ of the participants, and also the presence of association between nutrition intakes and 
diet variety (15). In another study conducted in Germany, the median intakes of dietary fibers, calcium, vitamin D and folate failed to reach two-third of the recommended amount in 4,020 free-living older men and women (16). Among the most widely studied bioactive micronutrients found in fruits and vegetables are carotenoids, flavonoids, organosulfurs, ascorbic acid, vitamin D, tocopherols, phenolic compounds and phytosterols (17). These compounds protect the body from inflammation, oxidative damage and accumulation of low-density lipoprotein cholesterol, and thereby prevent or slow down the progression of cardiovascular and metabolic diseases (17). Low serum micronutrients, such as serum carotenoids, tocopherols and 25-hydroxyvitamin D were independently associated with frailty among older women, and the risk of frailty increased with the number of micronutrient deficiencies (18). Coincidentally, fruit and vegetable intakes had been associated with greater bone mineral density in older adults (19). In view of the current evidence, our reported lack of fruit, vegetable and dairy intakes may have deleterious health effects in these older adults.

The World Health Organisation experts concluded that the proportion of Asian populations with a high risk of type 2 diabetes and cardiovascular disease is substantial at BMI lower than the existing WHO cut-off point for overweight $\left(25 \mathrm{~kg} / \mathrm{m}^{2}\right)$ (20). Although available data do not necessarily indicate a clear BMI cut-off point for all Asian for overweight or obesity, the cut-off point for the observed health risk varies from 22 to $25 \mathrm{~kg} / \mathrm{m}^{2}$ in different Asian populations (20). To align with the WHO guidelines, the Singapore Health Promotion Board set the BMI cut-off for moderate health risk at 23.0 and high risk at $27.5 \mathrm{~kg} / \mathrm{m}^{2}$. In the view that all our study participants are of Asian ethnicities and residing in Singapore, we interpreted and analysed our data using the Singapore Health Promotion Board's guideline on the BMI cut-off points. Average free-living older adults in Singapore are overweight and thereby exposed to moderate health risk, as indicated by their BMI, waist circumferences and waist-hip circumference ratios. Higher BMI and waist circumferences have been suggested to be strong indicators of health complications. BMI and waist circumferences were positively associated with systolic blood pressures, fasting plasma glucose and triglycerides, and inversely associated with HDL cholesterol (21). Higher body weight, BMI, waist circumferences, hip circumferences, waist-hip circumference ratios and visceral fat were strongly correlated with higher pulsewave velocity in 177 older adults $(22,23)$. The English Longitudinal Study of Ageing reported an association of frailty with high waist circumferences in older adults, suggesting that truncal obesity may be a target for intervention to improve mobility in older adults (24). Abdominal adiposity may be a stronger risk factor for chronic heart failure in older men and women as reported in a prospective, longitudinal study with 2435 older adults (25). High waist circumferences or triceps skinfold thickness increased risks of dementia in a recent meta-analysis (26). The same meta-analysis reported a significant U-shaped association between BMI and dementia with dementia risk increased for obesity and underweight (26). With the mounting amount of evidence associating obesity with physical function, quality of life and medical complications in older adults, weight loss may seems a necessity strategy to promote healthy living among our older adults. It is however important to ensure that the weight loss is not accompanied by muscle and bone mass loss in these older adults.

Our older adults have differing body fat distributions between the two genders. This is consistent with previous report that Asian males have lower body fat masses than their female counterparts (27). Males may be more likely to accumulate fatty tissues in their abdominal regions (as reflected by their significantly higher waist circumferences), which results in central adiposity. Females may be more likely to accumulate adipose tissues over many different body areas (as reflected in their significantly larger body fat mass percentage), which results in peripheral adiposity. Central adiposity, a form of obesity, is defined as the accumulation of abdominal fat resulting in an increase in waist size (28), and is becoming a major concern among Singaporean, including the older adult (29). The ageing process is usually accompanied by significant body composition change and deposition of fatty tissues around the abdominal area (30). Coincidentally, obesity-related diseases commonly appear at the second half phase of life which often burdens the older adults (31). High level of central adiposity has been associated with an increased risk level of number of metabolic diseases such as Type 2 diabetes mellitus, hypertension, heart disease and dementia (32). A recent meta-analysis reported significant correlations between body fat, BMI, waist circumferences and waist-hip circumference ratios in older adults (33). However, none of the studies included in this systematic review involves older adults of Asian ethnicity. Our study provides preliminary evidence of the significant correlations between body fat masses and obesity-related anthropometric measurements. More studies with larger numbers of older adults will be required to ascertain the relations between body fat masses with anthropometric measurements.

Our results were limited by the small sample size and uneven proportion of genders in the study group. It is, however, essential to note the significance of our results though they were based on a relatively small sample size when compared to other large cohort studies. The nutrition deficiency risk screening exercise should be expanded to include larger number of older adults According to the official Singapore population consensus, the proportion of gender in the age group between 50 and 80 years is approximately equal. It appears in our study that free-living women were more receptive towards 
health research study participation than their opposite gender in Singapore, even though a study of entire different scope will be required to examine this gender difference. The concept of nutrition risk is complex as nutrition status is influenced by a wide variety of psychosocial and biological factors, and screening for malnutrition in older people is recognized as difficult. The SCREEN II tool is essentially a risk evaluation for eating and nutrition. Screening identifies those at nutrition risk from established risk factors such as living alone and poor appetite so that a detailed nutritional assessment can be undertaken to measure food and nutrient intake. The SCREEN II tool is also not designed to examine the reasons behind the high risk, for example, why the older adults did not consume sufficient amount of fruits, vegetables and dairy products.

Alarmingly, majority of the participants were at high risk of nutrition deficiency and at the same time, had moderately high BMI. Nutrition deficiencies can be observed in normal-weight and overweight populations, largely due to unbalanced dietary intake. A recent US study reported a high prevalence of nutrition deficiency in overweight and obese compared to normal-weight individuals (34). The same study suggests that these overweight and obese individuals may consume an excess of dietary energy and yet may not meet their entire essential nutrition requirements (34). This trend may be prevalent in Asian countries like Singapore as their populations are used to consume energy dense diets. Older adults in the community tend to have significant nutrition concerns and deficits owing to the physiological, social, and psychological changes associated with aging. Nutrition offers the means to improve health and well-being when chosen carefully. Poor nutrition and eating habits should not be considered an inevitable consequence of aging. By correcting the eating habits and improving the nutritional status of older adults, significant health and quality of life gains can be achieved. In a placebo-controlled, randomized intervention study involving 101 older adults, participants in the milk supplemented group significantly increased their energy, protein, and essential vitamins and mineral intakes compared to the control group (35). Significant reduction in nutrition deficiency risk (or increase in SCREEN II score) was reported after an 18-month meal program in Canada (36). Nutrition deficiency risk assessment, such as the SCREEN II, and anthropometric measurements used in this study, promotes awareness and provides a base for nutrition behavioural changes (37). It also provides opportunities to improve nutrition of the screened population, and can be used to evaluate the effectiveness of a newly developed nutrition education program in free-living communities (37).

\section{Funding: The authors did not receive any funding for this study.}

Acknowledgement: The authors will like to thank the help rendered by the People's Association.
Conflict of interest: The authors do not have any conflict of interest.

Ethical standards: The study protocol has been approved by the Institutional Research Board, Nanyang Polytechnic, Singapore.

\section{References}

1. Christensen K, Doblhammer G, Rau R, Vaupel JW. Ageing populations: the challenges ahead. Lancet. 2009;374:1196-208.

2 McCullough ML, Feskanich D, Stampfer MJ, et al. Diet quality and major chronic disease risk in men and women: moving toward improved dietary guidance. Am J Clin Nutr. 2002;76:1261-71.

3 Nocon M, Hiemann T, Müller-Riemenschneider F, Thalau F, Roll S, Willich $\mathrm{SN}$. Association of physical activity with all-cause and cardiovascular mortality: a systematic review and meta-analysis. Eur J Cardiovasc Prev Rehab. 2008;15:239-46.

4 Burke L, Jancey J, Howat P, et al. Physical activity and nutrition program for seniors (PANS): protocol of a randomized controlled trial. BMC Pub Health. 2010;10:751.

5 Holland CA, Everitt P, Johnson A, Devi R. The 'Healthy Passport'intervention with older people in an English urban environment: effects of incentives and peer-group organisers in promoting healthy living. Ageing Soc. 2008;28:525-49.

6 Koh G-H, Tai B, Ang L-W, Heng D, Yuan J-M, Koh W-P. All-cause and cause-specific mortality after hip fracture among Chinese women and men. Osteopor Int. 2013;24:1981-9.

7 Seow A, Koh W-P, Wang R, Lee H-P, Yu MC. Reproductive Variables, Soy Intake, and Lung Cancer Risk among Nonsmoking Women in the Singapore Chinese Health Study. Cancer Epidemiol Biomark Prev. 2009;18:821-7.

8 Teng GG, Ang L-W, Saag KG, Mimi CY, Yuan J-M, Koh W-P. Mortality due to coronary heart disease and kidney disease among middle-aged and elderly men and women with gout in the Singapore Chinese Health Study. Ann Rheum Dis. 2012;71:924-8.

9 Brownie S. Why are elderly individuals at risk of nutritional deficiency? Int J Nurs Prac. 2006;12:110-8.

10 Evidence based practice guidelines for the nutritional management of malnutrition in adult patients across the continuum of care. Nutr Dietet. 2009.

11 Mahan LK, Escott-Stump S, Raymond JL. Krause's food \& the nutrition care process: Elsevier Health Sciences; 2012.

12 Keller H, Goy R, Kane S. Validity and reliability of SCREEN II (Seniors in the community: risk evaluation for eating and nutrition, Version II). Eur J Clin Nutr. 2005;59:1149-57.

13 Phillips MB, Foley AL, Barnard R, Isenring EA, Miller MD. Nutritional screening in community-dwelling older adults: a systematic literature review. Asia Pac J Clin Nutr. 2010;19:440.

14 Wham CA, Teh R, Robinson M, Kerse N. What is associated with nutrition risk in very old age? J Nutr Health Aging. 2011;15:247-51.

15 Marshall TA, Stumbo PJ, Warren JJ, Xie X-J. Inadequate Nutrient Intakes Are Common and Are Associated with Low Diet Variety in Rural, CommunityDwelling Elderly. J Nutr. 2001;131(8):2192-6.

16 Volkert D, Kreuel K, Heseker H, Stehle P. Energy and nutrient intake of young-old, old-old and very-old elderly in Germany. Eur J Clin Nutr. 2004;58:1190-200.

17 Dauchet L, Amouyel P, Dallongeville J. Fruits, vegetables and coronary heart disease. Nat Rev Cardiol. 2009;6(9):599-608.

18 Semba RD, Bartali B, Zhou J, Blaum C, Ko C-W, Fried LP. Low Serum Micronutrient Concentrations Predict Frailty Among Older Women Living in the Community. J Gerontol Ser A: Biol Sci Med Sci. 2006;61:594-9.

19 Tucker KL, Hannan MT, Chen H, Cupples LA, Wilson PW, Kiel DP. Potassium, magnesium, and fruit and vegetable intakes are associated with greater bone mineral density in elderly men and women. Am J Clin Nutr. 1999;69(4):727-36.

20 Nishida C, Consultation WE. Appropriate body-mass index for Asian populations and its implications for policy and intervention strategies. Lancet. 2004;363:157-63.

21 Abbasi F, Blasey C, Reaven GM. Cardiometabolic risk factors and obesity: does it matter whether BMI or waist circumference is the index of obesity? Am J Clin Nutr. 2013 September 1, 2013;98:637-40.

22 Wildman RP, Mackey RH, Bostom A, Thompson T, Sutton-Tyrrell K. Measures of Obesity Are Associated With Vascular Stiffness in Young and Older Adults. Hypertension. 2003;42:468-73.

23 Sutton-Tyrrell K, Newman A, Simonsick EM, et al. Aortic Stiffness Is Associated With Visceral Adiposity in Older Adults Enrolled in the Study of Health, Aging, and Body Composition. Hypertens. 2001;38:429-33.

24 Hubbard RE, Lang IA, Llewellyn DJ, Rockwood K. Frailty, Body Mass Index, and Abdominal Obesity in Older People. J Gerontol Ser A: Biol Sci Med Sci. 2010;65A:377-81.

25 Nicklas BJ, Cesari M, Penninx BW, et al. Abdominal obesity is an independent risk factor for chronic heart failure in older people. J Am Geriatr Soc. 2006; $54: 413-20$. 
26 Beydoun $\mathrm{M}$, Beydoun $\mathrm{H}$, Wang Y. Obesity and central obesity as risk factors for incident dementia and its subtypes: a systematic review and metaanalysis. Obes Rev. 2008;9:204-18.

27 Wang J, Thornton JC, Russell M, Burastero S, Heymsfield S, Pierson RN Asians have lower body mass index (BMI) but higher percent body fat than do whites: comparisons of anthropometric measurements. Am J Clin Nutr. 1994;60:23-8.

28 Despres J-P, Lemieux I. Abdominal obesity and metabolic syndrome. Nature. 2006;444:881-7.

29 Ford ES, Mokdad AH, Giles WH. Trends in Waist Circumference among U.S. Adults. Obesity Res. 2003;11:1223-31.

30 Wannamethee S, Shaper A, Lennon L. REasons for intentional weight loss, unintentional weight loss, and mortality in older men. Arch Int Med. 2005; $165: 1035-40$.

31 Thompson D, Edelsberg J, Colditz GA, Bird AP, Oster G. LIfetime health and economic consequences of obesity. Arch Int Med. 1999;159:2177-83.

32 Han TS, Tajar A, Lean MEJ. Obesity and weight management in the elderly. Br Med Bullet. 2011;97:169-96.
33 McTigue KM, Hess R, Ziouras J. Obesity in older adults: a systematic review of the evidence for diagnosis and treatment. Obesity. 2006;14:1485-97.

34 Kimmons JE, Blanck HM, Tohill BC, Zhang J, Khan LK. Associations Between Body Mass Index and the Prevalence of Low Micronutrient Levels Among US Adults. Medscape General Medicine. 2006 12/19;8(4):59-60.

35 Barr SI, McCarron DA, Heaney RP, et al. Effects of Increased Consumption of Fluid Milk on Energy and Nutrient Intake, Body Weight, and Cardiovascular Risk Factors in Healthy Older Adults. J Am Dietet Assoc. 2000;100:810-7.

36 Keller HH. Meal Programs Improve Nutritional Risk: A Longitudinal Analysis of Community-Living Seniors. J Am Dietet Assoc. 2006;106:1042-8.

37 Wijnhoven HAH, Schilp J, van Bokhorst-de van der Schueren MAE, et al Development and validation of criteria for determining undernutrition in community-dwelling older men and women: The Short Nutritional Assessment Questionnaire 65+. Clin Nutr. 2012;31:351-8. 